\title{
Securing clinic tele-diagnostic system using enhanced tiny encrypted radio frequency identification and image steganographic technique
}

\author{
Olayemi M. Olaniyi* \\ Department of Computer Engineering, \\ Federal University of Technology, \\ P.M.B. 65, Minna, Niger-State, Nigeria \\ Email: mikail.olaniyi@futminna.edu.ng \\ *Corresponding author \\ Oladiran T. Arulogun \\ Department of Computer Science and Engineering, \\ Ladoke Akintola University of Technology, \\ P.M.B. 4000, Ogbomoso, Oyo State, Nigeria \\ Email: otarulogun@lautech.edu.ng \\ Adebayo Omotosho \\ Department of Computer Science, \\ Landmark University, \\ P.M.B. 1004, Omu-Aran, Kwara State, Nigeria \\ Email: omotosho.adebayo@Imu.edu.ng \\ Victor O. Onuh \\ Department of Computer Engineering, \\ Federal University of Technology, \\ P.M.B. 65, Minna, Niger-state, Nigeria \\ Email: victor.onuh@st.futminna.edu.ng
}

\begin{abstract}
Traditional health record systems are gradually giving way for automated solutions capable of delivery robust e-healthcare systems. Existing models of e-healthcare system carries with it challenges of privacy breach in electronic health record (EHR) authentication system. The existence of patients' private data within the channel of communication could be intercepted, interpreted and used fraudulently leading to loss of data confidentiality. Also, an unprotected RFID tag in EHR system could be cloned and impersonated thus, depriving the patients of guaranteed privacy. In this paper, we present a cryptographic approach for securing data communications in clinic tele-diagnostic system (CTDS). Analysis of the performance of the system showed an imperceptible stego image with peak signal to noise ratio greater than $30 \mathrm{db}$. Furthermore, valid patient's RFID tag was authenticated with the developed pseudo-random tiny encryption-based RFID-EHR system. The performance evaluation of the system portrays a system capable of counteracting the effects of tag cloning, location tracking and replay attacks in data communication channels of clinic tele-consultations.
\end{abstract}

